Revue d'histoire de l'Amérique française

REVUE D.HISTOIRE DE L'AMÉRIQUE FRANÇAISE

\title{
La contribution scientifique de La Galissonnière au Canada
}

\section{Roland Lamontagne}

Volume 13, numéro 4, mars 1960

URI : https://id.erudit.org/iderudit/302005ar

DOI : https://doi.org/10.7202/302005ar

Aller au sommaire du numéro

Éditeur(s)

Institut d'histoire de l'Amérique française

ISSN

0035-2357 (imprimé)

1492-1383 (numérique)

Découvrir la revue

Citer cet article

Lamontagne, R. (1960). La contribution scientifique de La Galissonnière au

Canada. Revue d'histoire de l'Amérique française, 13(4), 509-524.

https://doi.org/10.7202/302005ar d'utilisation que vous pouvez consulter en ligne.

https://apropos.erudit.org/fr/usagers/politique-dutilisation/ 


\section{LA CONTRIBUTION SCIENTIFIQUE DE LA GALISSONIËRE AU CANADA *}

Le gouverneur intérimaire de la Nouvelle-France, RolandMichel Barrin, comte de La Galissonière, ${ }^{1}$ a participé à l'activité scientifique de son époque. Il a communiqué avec des hommes de science éminents tels que Henri-Louis Duhamel du Monceau, Pierre Bouguer, Louis-Guillaume Lemonnier, Nicolas-Louis de Lacaille, Jean-Étienne Guettard.

Un opuscule intitulé Avis pour le transport par mer des arbres, des plantes vivaces, des semences, des animaux et de différents autres morceaux d'histoire naturelle (s.l., 1752) est attribué à Duhamel du Monceau et à Roland-Michel Barrin de La Galissonière. ${ }^{2}$ Ce dernier en faisait tenir copie à GaspardJoseph Chaussegros de Léry: "Vous faittes très bien de ramasser

* L'auteur remercie M. Pierre Brunet, adjoint de l'Archiviste du Canada, M. J.-Antoine Pelletier, bibliothécaire adjoint des Archives de la Province de Québec, M. Yves Laissus, archiviste-paléographe du Muséum national d'histoire naturelle de Paris, M. J. P. Hudson, conservateur adjoint du département des manuscrits du British Museum, qui lui ont fait parvenir des photocopies et microfilms de pièces intéressant le comte de La Galissonnière. Il tient à exprimer sa gratitude à $M$. Louis de Broglie et à $\mathbf{M}$. R. Courrier, secrétaires perpétuels de l'Académie des Sciences, à M. le Capitaine de Vaisseau Rostand, chef du Service historique du Ministère des Armées (Marine), à M. l'Ingénieur Général Gougenheim, directeur du Service hydrographique de la Marine, à M. Jacques Lévy, astronome à l'Observatoire de Paris, à M. Joseph-R. Leduc, conservateur de la Bibliothèque centrale de l'Université de Montréal et à $M^{\text {lle }}$ Claire Audet ainsi qu'à M. James J. Heslin, directeur adjoint de la New York Historical Society pour les renseignements qu'ils lui ont communiqués.

L'auteur serait très reconnaissant à ceux qui posséderaient d'autres sources manuscrites relatives à l'activité scientifique de Roland-Michel Barrin de La Galissonière de lui transmettre les cotes des manuscrits.

1 La Galissonière porte parfois le titre de marquis. Voir Guy Frégault, François Bigot, administrateur français (2 vol., Montréal, 1948), I: 300 (note 61).

2 La Bibliothèque Saint-Sulpice de Montréal conserve une copie de la première édition de cet opuscule. La Bibliothèque Nationale de Paris possède deux exemplaires de la première édition de cet opuscule. In-12, 55 p. ainsi que deux exemplaires de la seconde édition. In-12, xii-90 p. (Imprimerie Royale, Paris, 1753). Catalogue général des livres imprimés de la Bibliothèque Nationale (Paris, 1925), tome LXXXVI, 125-126. 
des plantes, noiaux et autres semences qui pourront être utiles en Canada je vous envoie un livret qui vous enseignera les moyens de les Conserver. ${ }^{3}$

Roland-Michel Barrin de La Galissonière fut élu Associé libre de l'Académie Royale des Sciences en 1752 et mourut en 1756. Entre ces limites chronologiques, selon les Secrétaires perpétuels de l'Académie des Sciences, aucun rapport de La Galissonière ne se trouve dans les registres de cette époque. ${ }^{4}$ Le Traité des arbres et arbustes de Duhamel du Monceau atteste l'apport de La Galissonière. ${ }^{5}$

Ce modeste inventaire de travaux scientifiques de La Galissonière doit être complété par l'étude des sources manuscrites. Le journal de bord du Héros contient un relevé d'observations relatives à l'emploi d'un «nouvel instrument pour prendre [la] hauteur $\gg .6$ La correspondance entre La Galissonière et Duhamel du Monceau a été colligée par Claude de Bonnault d'après les pièces conservées aux Archives du Château de Denainvilliers; les copies dactylographiées ont été déposées aux Archives Publiques $d u$ Canada. ${ }^{7}$ Les documents de Lotbinière, dont les photocopies de manuscrits provenant de la New York Historical Society sont conservées aux Archives Publiques du Canada, laissent supposer que les rapports épistolaires ont dû être fréquents entre La Galis-

3 La Galissonière, Paris, 15 juillet 1752. Archives de la Province de Québec.

${ }^{4}$ L'Histoire de l'Académie Royale des Sciences ne contient qu'une mention, année 1755 , p. 39. En voici le texte: \& M. le Marquis de La Galissonière a fait voir à l'Académie des morceaux d'une espèce de granit trouvé près de Montaigu, et qui est bien susceptible du plus beau poli. Il est étonnant de voir combien cette espèce de pierre, que l'on croyait propre à la Haute-Egypte, est commune dans le Royaume. 》

5 Henri-Louis Duhamel du Monceau, Traité des arbres et arbustes qui se cultivent en France en pleine terre (2 vol., Paris, 1758). Voir Claude de Bonnault, «La Galissonière et sa contribution à la botanique du Canada », Les Botanistes français en Amérique du Nord avant 1850. Colloques internationaux du Centre national de la recherche scientifique (Paris, 1956), 175. ${ }_{6}$ «Journal de bord. Deux pièces, $1^{\text {ere }} L e V^{a u}$ Le heros 1737. Commd M. De la Galissonière de l'Ile Daix a Louisbourg et retour a L'Ile d'Aix. $2^{\text {de }}$ Extrait et depouillement du journal. 》 Archives de la Marine, 4 J.J., liasse 8, no 50. Transcription conservée aux Archives Publiques du Canada. (M. G. [Manuscript Group] 2/9). A l'avenir: Journal de bord du Héros. $A P C$.

7. G. 18, G 11, 33 p. Archives Publiques du Canada. A l'avenir: 
sonière et Michel Chartier, marquis de Lotbinière. ${ }^{8}$ Le manuscrit de la Flore de Jean-François Gaulthier, ${ }^{9}$ des lettres et un mémoire d'instruction de La Galissonière à Chaussegros de Léry se trouvent aux Archives de la Province de Québec. La Bibliothèque centrale du Muséum national d'histoire naturelle possède quatre pièces intéressant La Galissonière dont $M$. Yves Laissus rapporte, en quelques lignes pour chaque lettre, des indications sur leur contenu. ${ }^{10}$ La correspondance privée fournit à l'étude de la politique coloniale de La Galissonière un indispensable supplément aux Archives des Colonies et des Affaires Étrangères. Elle est peu nombreuse. Il est permis de supposer que les dépôts d'archives privées et publiques de France en recèlent davantage. ${ }^{11}$

Barrin de La Galissonière s'intéressait aux mathématiques..$^{12}$ Ses échanges scientifiques avec le professeur d'hydrographie, le Père Pierre-Joseph de Bonnécamps, S.J., Gaspard-Joseph Chaus-

8 APC. Papiers de Lotbinière, No 1-25, 1, 1663-1753, M. G. 18, K 3, 2 (1) et No 27-105, 2, 1753-1759, M. G. 18, K 3,2(2).

9 Jacques Rousseau, «Michel Sarrazin, Jean-François Gaulthier et l'étude pré-linnéenne de la flore canadienne», Les Botanistes français en Amérique du Nord avant 1850, 149-157.

10 1. Lettre autographe signée à Duhamel du Monceau. $A$ bord du Tigre, en rade de Toulon, $7-8$ octobre 1741 . Cette lettre aborde successivement divers sujets: améliorations portuaires en France, remerciements pour les Elémens de géographie dont il est très content et qu'il a prêté au Père Châtelard, un long développement sur de futurs ou d'éventuels envois de graines au Jardin du roi.

2 et 3 . Deux lettres autographes signées à Lemonnier, Fontainebleau, 5 novembre 1751 et Paris, $1^{\text {er }}$ octobre 1752 . Lettres relatives à des envois de graines et semences et à l'identification difficile de certaines.

4. "Noms de quelques arbres, arbrisseaux et plantes étrangères que Monsieur le marquis de La Galissonière fait cultiver à son château de la paroisse de Monnière environ 4 lieues de la ville de Nantes ». (s.l.n.d.).

11 J.-Edmond Roy, Rapport sur les archives de France relatives d̀ l'histoire du Canada (Ottawa, 1911), 1029.

12 La Galissonière propose une solution mathématique à un problème de construction navale. "Peut être que la difficulté de la construire [la courbe de L'Hospital] a retenu plus qu'autre chose ceux qui auroient voulu s'en servir ou du moins en approcher. Ce que je demanderois seroit donc qu'en divisant l'axe en un grand nombre de parties, à commencer du sommet jusqu'à cinquante ou soixante pieds, on dît: à un pied du sommet la courbe a ... d'ouverture; à deux pieds, elle a ... d'ouverture, et ainsi du reste. Nous pourrions voir alors, en quelque façon pour nos yeux si on doit désespérer, comme semble faire $M$. de Fontenelle de marier cette sublime théorie avec ce que nous pourrions nous dispenser de conserver de nos vieilles rubriques. 》 La Galissonière à Duhamel du Monceau, Rochefort, 7 mars 1739. $A P C$. (M. G. 18, G. 11), 1. 
segros de Léry, Michel Chartier de Lotbinière et Jean-François Gaulthier montrent l'influence heureuse que La Galissonière a exercé sur les chercheurs du Canada au XVIII ${ }^{e}$ siècle.

A son retour d'un voyage à Québec en 1739 , La Galissonière recommandait à Duhamel du Monceau un botaniste canadien :

L'abbé Gosselin mériteroit qu'on fît quelque chose pour lui, car il se donne bien de la peine. Il n'est non plus que moi grand botaniste, mais on aura plus de plantes par son moien que par quelqu'un beaucoup plus habile mais qui seroit moins laborieux et moins infatigable. Il n'en coûteroit rien au Roi pour le mettre un peu à son aise et plus en état de travailler utilement. ${ }^{13}$

Dès 1739, La Galissonière cherchait à stimuler le développement scientifique et technique de la colonie. "Le mémoire sur la potasse, écrit-il à Duhamel du Monceau, peut être utile au Canada où le hêtre abonde partout, mais il faudroit qu'un habile homme comme vous rectifiât la méthode qui y est indiquée et en donnât une pour fabriquer de la potasse aisément et à bon marché. ${ }^{14}$ Le ministre de la Marine écrivait au marquis de La Jonquière, le 4 mai 1749: «Monsieur de La Galissonière a proposé d'établir une imprimerie dans la colonie: laquelle il a représentée devoir y être d'une grande utilité pour la publication des ordonnances et des règlements de police ... le Roi ne jugeant pas à propos de faire la dépense d'un pareil établissement, il faut attendre que quelque imprimeur se présente pour y pourvoir et dans ce cas j'examinerai à quelles conditions il pourra convenir de lui donner un privilège. $\gg^{15}$

Autant qu'il le peut, si l'on en juge d'après sa correspondance, La Galissonière se tient au courant des publications scientifiques. Il lit les Mémoires de l'Académie Royale des Sciences, les ouvrages de Pierre Bouguer sur l'astronomie et de Cassini de Thury sur la carte de France, ${ }^{16}$ ainsi que les mémoires de Buffon

13 Id. à $I d$., $A$ bord $d u R u b i, 12$ décembre 1739. Ibid., 4.

14 Id. à Id., Rochefort, 3 avril 1739 . Ibid., 3.

15 Cité par Pierre-Georges Roy, «L'imprimerie dans la NouvelleFrance », Bulletin des recherches historiques, 10 (1904), 190.

16 Cassini de Thury commença cette carte qui fut terminée par son fils en 1815. C'est la première carte topographique d'un grand pays qui ait été dessinée suivant des méthodes scientifiques. Maurice Daumas, Histoire de la Science (Paris, 1957), 749. 
et de Duhamel du Monceau. Il envoie à Chaussegros de Léry la Connoissance des tems ${ }^{17}$ qu'il destine au Père Bonnécamps dont il reconnaît les mérites scientifiques. Il recommandait à Lotbinière de soumettre les résultats d'observation au savant jésuite. «En cas que je sois party je vous prie de repasser avec le R. P. Bonecamp les diverses observations de latitude que vous aurés faites et de me les envoier ».18 La Galissonière expédie à l'ingénieur du roi Lotbinière l'ouvrage de Alexandre-Guy Pingré, l'État $d u$ ciel pour l'année 1755. Il lui avait annoncé l'envoi d'une liste de livres et d'instruments qui ne figure pas parmi les pièces de cette correspondance:

J'ai obtenu aujourd'hui de M. Rouillé [ministre de la Marine] ${ }^{19}$ des instruments pour votre usage et j'espère en obtenir aussi des livres; je vous envoierai la liste des uns et des autres; mais je ne sai si vous aurés les instruments, au moins tous ceux que je voudrois que vous eussiés; ils seront cependant commandés dès demain ».

Dans la même lettre, Barrin de La Galissonière insiste sur la difficulté d'obtenir des instruments de physique. ${ }^{20}$ Il avait stimulé Chaussegros de Léry à se perfectionner dans le domaine des sciences appliquées. "Vous avés très bien fait aussi de vous appliquer pendant votre séjour à la Rochelle à approfondir le nouvel exercice et les évolutions mais cela ne doit pas vous Em-

${ }^{17}$ La Galissonière à Chaussegros de Léry, Paris, 15 juillet 1752. Archives de la Province de Québec.

La Connaissance des temps au méridien de Paris, publié par ordre de l'Académie Royale des Sciences donnait chaque année, depuis 1679 , de nombreuses tables sur les mouvements des astres, suivies de notices explicatives. Roger Heim, éd., Buffon. Collection des Grands Naturalistes Français, (Paris, 1952), 192.

18 La Galissonière à Lotbinière, Québec, 12 juillet 1749. APC. Papiers de Lotbinière, No 1-25, 1, 1663-1753, M. G. 18, K 3, 2 (1).

19 Louis-Antoine Rouillé, comte de Jouy, avait succédé à Jean-Frédéric Phélypeaux, comte de Maurepas, exilé le 24 avril 1749. Guy Frégault, Le Grand Marquis, Pierre de Rigaud de Vaudreuil et la Louisiane (Montréal, 1952), 285. Voir au sujet des circonstances de la disgrâce de Maurepas, Id., François Bigot, administrateur français, I: $356 \mathrm{~s}$.

20 «On ne renvoie pas la monture du Globe d'Electricité que vous m'avés laissée, parce qu'ainsi que vous l'avés marqué sur le papier qui l'enveloppe, il faudroit envoyer en même tems une machine pneumatique et comme vous savés qu'on n'a pas ce qu'on veut, j'aime mieux vous envoyer des instrumens utiles. » La Galissonière à Lotbinière, Paris, 19 février 1754 . APC. Papiers de Lotbinière, No 27-105, 2, 1753-1759, M. G. 18, K 3, 2 (2). 
pêcher de continuer à cultiver la partie du génie dans laquelle vous pouvés beaucoup vous perfectionner sous M. votre pere et par le travail des Fortifications qui se fait à Québec. »21

Lotbinière avait reçu du ministre Rouillé une « bourse d'étude »; La Galissonière lui écrivait, le 27 septembre 1752 :

Le Longtemps qui s'est passé, monsieur, avant que je puisse vous remettre le petit fond qui vous a été destiné pour vous et pour vos camarades de voiage et d'étude, me fait un peu douter si vous aurés assez de temps et d'argent pour exécuter les vües du ministre, qui m'a chargé de vous les expliquer; mais qui au surplus n'ignore pas Combien la saison est avancée, et qui souhaite que vous reveniés de bonne heure à Paris pour y reprendre vos études Commencées ou en Commencer d'autres $\gg .^{22}$

Lotbinière avait accompli une mission technique au Luxembourg. ${ }^{23}$ Il ira plus tard visiter les forges et la manufacture d'armes à Angoulême. ${ }^{24}$

La Galissonière consacre ses loisirs à l'observation directe des faits en histoire naturelle. A son château de la paroisse de Monnière, près de Nantes, il fait cultiver des arbres, des arbrisseaux et des plantes exotiques ${ }^{25}$ à l'exemple du roi et des grands seigneurs dont les domaines devenaient de véritables jardins botaniques. ${ }^{26}$ «Quand je serai débarrassé du vaisseau, écrit-il à

21 La Galissonière à de Léry, Paris, 15 juillet 1752. Archives de la Province de Québec.

22 Id. à Lotbinière, Paris, 27 septembre 1752. APC. Papiers de Lotbinière, No 1-25, 1, 1663-1753, M. G. 18, K 3, 2 (1).

23 « Vous devés bien Compter ne pas voir Luxembourg bien à votre aise, et vous ne devés pas mesme témoigner trop d'empressement pour qu'on vous montre que ce qu'on voudra bien vous montrer; mais au moyen de la vrécantion que vous prenés d'étudier le plan vous en verrés plus d'un coup d'œil que d'autres ne feroient par un long examen.»Id. à Id., Paris, 22 juillet 1752 . ibid.

24 Id. à Id., Paris, 12 novembre 1753. APC. Papiciss de Lotbinière, No 27-105, 2, 1753-1759, M. G. 18, K 3, 2 (2).

25 « Noms de quelques arbres, arbrisseaux, et plantes étrangères que Monsieur le marquis de La Galissonière fait cultiver à son château de la paroisse de Monnière environ 4 lieues de la ville de Nantes ». (s.l.n.d.). Bibliothèque centrale du Muséum national d'histoire naturelle.

26 A. Guillaumin et V. Chaudin, \& L'introduction en France des plantes horticoles originaires de l'Amérique du Nord avant $1850 \gg$, Les Botanistes français en Amérique du Nord avant 1850, 123. 
Duhamel du Monceau, et que je verrai jour à aller faire un tour chez moi, je vous demanderai aussi du plant ou des greffes de quelques espèces d'arbres fruitiers que vous avés et qui me manquent. ${ }^{27}$ Il semble que l'on décèle un peu d'amertume dans cette lettre du 2 décembre 1742: "Comme vous connaissés mon goût et ma situation et que vous savés que je n'ai ni la volonté ni le pouvoir d'avoir un jardin botanique en forme, j'espère que vous ne ferés pas de difficulté, non plus que Monsieur de Jussieu de prendre tout ce qui conviendra pour vous ou pour vos amys. $\gg 28$ C'est avec une pointe d'humour que La Galissonière avouera plus tard: «Toutes ces absences ont sans doute beaucoup gâté mon jardin et peut-être mon jardinier. C'est ce qui fait que de tant de choses que vous lui avés envoiées ou que j'ai envoiées de mon côté, il en a si peu conservé. ${ }^{29}$

Au cours de ses voyages, La Galissonière recueille des échantillons d'ornythologie, ${ }^{30}$ de conchyliologie, de biologie marine ${ }^{31}$ et de botanique qu'il fait parvenir au Jardin royal de Rochefort ou de Paris, à Jussieu, à Lemonnier et à Duhamel du Monceau. Il veut obtenir, entre autres, un arbre comme celui qu'il a vu «chez M. de Longueuil à Ste Hélène, près de Montréal », un érable piqueté qu'il qualifie de «fort rare», des petites poires sauvages qui se trouvent chez l'abbé La Corne, curé de La Durantaie. $^{32}$ Il envoie des plantes du Canada à M. du Puy pour le Jardin de Rochefort. ${ }^{33}$ Il introduit en France le magnolia à grandes fleurs. ${ }^{34}$ Il expédie de grandes quantités de noyaux de cerise au Canada et il encourage Lotbinière à recueillir des échantillons de matières botaniques..$^{35}$ Il avait annoté le manuscrit de la Flore de Jean-François Gaulthier. Ce dernier incitait le naturaliste

${ }^{27} \mathrm{La}$ Galissonière à Duhamel du Monceau, $A$ bord $d u R u b i, 12$ décembre 1739. $A P C$. (M. G. 18, G. 11), 5 .

28 Id. à Id., Toulon, 2 décembre 1742. Ibid., 12.

29 Id. à Id., Brest, 3 février 1745. Ibid., 27.

30 Id. à Id., $A$ bord du Rubi, 12 décembre 1739. Ibid., 5.

31 Id. à Id., Toulon, 4 avril 1743. Ibid., 24.

32 Id. à Id., Toulon, 6 décembre 1742. Ibid., 15.

33 Id. à Id., Rochefort, 3 avril 1739 . Ibid., 3. XXVIII.

34 Les Botanistes français en Amérique du Nord avant 1850, planche

35 La Galissonière à Lotbinière, Paris, 22 juillet 1752. APC. Papiers de Lotbinière, No 1-25, 1, 1663-1753, M. G. 18, K 3, 2 (1). 
Jean-Etienne Guettard ${ }^{36}$ à demander conseil à Barrin de La Galissonière. ${ }^{37}$

$\mathrm{Au} \mathrm{XVIII}$ siècle, l'acclimatation des plantes étrangères constituait une partie intégrante de la politique coloniale de la métropole. ${ }^{38}$ En 1726, une ordonnance du roi assujettit les capitaines nantais « à rapporter des pays étrangers et des colonies françaises les graines et les plantes nécessaires au Jardin des plantes médicinales établi à Nantes (depuis 1688) et oblige les apothicaires de cette ville à en approvisionner constamment le Jardin Royal de Paris ${ }^{39}$ La correspondance de Bernard de Jussieu avec le botaniste Prat en Louisiane montre l'attention qu'apportait aux plantes le ministre de Louis XV, Jean-Frédéric Phélypeaux, comte de Maurepas. ${ }^{40}$

Barrin de La Galissonière a établi des échanges scientifiques, non pas uniquement avec les hommes de science, mais aussi avec les administrateurs, l'intendant de la Nouvelle-France Gilles Hocquart, les commandants des forts, notamment Paul-Louis de Lusignan, commandant du Fort Saint-Frédéric et Daniel-Hyacinthe Liénard de Beaujeu, commandant du Fort Niagara. Il a obtenu la collaboration du fournisseur du roi aux colonies, Abraham Gradis, négociant de Bordeaux, d'origine juive.

L'intendant Hocquart avait déjà expédié des échantillons de matières botaniques au Jardin du Roi. ${ }^{41}$ Il avait demandé au ministre des instruments scientifiques, une pendule à seconde et une lunette montée sur un quart de cercle qui étaient destinés

36 Douglas McKie, Antoine Lavoisier, Scientist, Economist, Social Reformer (Londres, 1952), 63-71. - Jean-Etienne Guettard herborisait en compagnie de René-Antoine Ferchault de Réaumur. Ad. Davy de Virville, 《Réaumur botaniste 》, Revue d'histoire des sciences et de leurs applications, XI (1958), 134.

37 Lettre de Gaulthier à Guettard, 21 octobre 1752, citée par Mme Gabrielle Duprat, « Essai sur les sources manuscrites conservées au Muséum national d'histoire naturelle», Les Botanistes français en Amérique du Nord avant 1850, 233.

38 Gilbert Chinard, «Les Michaux et leurs précurseurs », Ibid., 272.

39 G. Duprat, « Essai sur les sources manuscrites conservées au Muséum national d'histoire naturelle $\gg$, Ibid., 238.

40 Jean F. Leroy, « Note sur l'introduction des plantes américaines en France dans la première moitié du XVIII ${ }^{e}$ siècle », Ibid., 285.

41 Douglas Brymner, Rapport sur les Archives du Canada pour l'année 1887 (Ottawa, 1888), cxv. 
au Père Bonnécamps, S.J. Il présentait un projet d'observatoire. ${ }^{42}$ Par l'intermédiaire de Gilles Hocquart, La Galissonière avait été mis au courant de l'existence du «pin-cyprès qui diffère du pin rouge en ce que les feuilles de ceux-ci viennent deux à deux sur un pédicule et celle du pin-cyprès trois à trois ... Je ferois plus de cas de cette découverte que celle d'une mine d'or, car il y en a abondamment. Cela meriteroit bien d'être suivi ainsi que les chesnes verds du Mississipi $\gg .^{43}$

Le commandant du Fort Saint-Frédéric, Paul-Louis de Lusignan avait montré à Pierre Kalm le document que recevaient les commandants des forts en vue d'établir un inventaire d'histoire naturelle dans les régions soumises à leur juridiction. Selon le botaniste Kalm, ce mémoire avait été rédigé par Jean-François Gaulthier et corrigé par Barrin de La Galissonière. ${ }^{44}$

Le commandant du Fort Niagara, Daniel-Hyacinthe Liénard de Beaujeu, écrit au gouverneur qu'il se conformera autant qu'il le pourra aux articles du «mémoire des plantes et graines pour les faire ramasser en temps convenable ».45 Il lui fait parvenir des échantillons de matières botaniques et de coquilles. ${ }^{46}$ Il signa-

42 Ibid., cxxx. - Dans une lettre de Paris, le 22 janvier 1755, adressée à Lotbinière, Pierre Bouguer se référait à la pendule du Père Bonnécamps. \& L'allongement qu'il vous a fallu donner à la verge de votre pendule pour luy faire battre les secondes de temps moyen fournieroit un fait très singulier, s'il n'y avoit tout lieu de croire qu'on s'est trompé à Paris en marquant un repaire. Je ne doute pas qu'il n'y ait quelquefois des circonstances locales qui changent la longueur du pendule, mais il est bien difficile de penser que la différence puisse aller à une ligne. S'il en est encore temps, je recommanderai de marquer avec soin le repaire sur la pendule du Pere Bonecamp et je me chargerai même bien volontiers de vérifier, si on y a réussi 》. APC. Papiers de Lotbinière, No 27-105, 2, 1753-1759, M. G. 18, K 3, 2 (2).

43 La Galissonière à Duhamel du Monceau, Brest, 3 février 1743. APC. (M. G. 18, G. 11), 17.

44 This useful paper was drawn up by the order of the marquis La Galissonière by $M$. Gaulthier, the royal physician at Québec and afterwards corrected and improved by the marquis 's own hand. He had several copies made of it, which he sent to all officers in the forts, and likewise learned men who travelled in the country. $\gg$ Peter Kalm, Travels into North America (2 vol., Londres, 1772). Traduction de John Reinhold Forster, 2: 184-185.

45 \& Lettres de Daniel-Hyacinthe Liénard de Beaujeu, commandant au fort Niagara à M. de La Galissonière », Bulletin des recherches historiques, (1931), 358.

46 Ibid., 357. 
le les difficultés relatives à la récolte d'échantillons: «On vous dit, Monsieur, qu'il est aisé de trouver toutes ces plantes, mais point du tout, elles ne sont ni communes ni près de Niagara, et je n'ai personne propre à pareille recherche, malgré cela vous pouvez conter (sic) que je ne me rebuterai point. ${ }^{47}$ Liénard de Beaujeu ajoute qu'il parcourra tous les mémoires de La Galissonière «pour faire ramasser à propos les plantes et graines de nos quartiers ».

Le fournisseur du roi aux colonies, Abraham Gradis, collabore à l'œuvre de La Galissonière «pour garnir le Canada de fruits d'Europe »,48 sans réclamer de frais d'expédition; La Galissonière lui en exprime sa gratitude. ${ }^{49}$ Ce n'est pas dans le simple but de stimuler le négociant Gradis qu'il écrivait, le 20 mai 1754: "Je vous remercie de l'envoi de mes graines en Canada et de celles que vous y avés jointes. Il fait toujours bon procurer de petits agrémens à cette colonie en attendant que par ses forces ou par la protection du Roi elle acquière des avantages plus solides et plus importans, c'est-à-dire qu'elle se mette en état de fournir plus d'objets au commerce $\gg .^{50}$ Nous verrons plus loin que cette activité botanique s'insérait dans le cadre de l'œuvre de politique coloniale de La Galissonière.

La curiosité scientifique de La Galissonière n'est pas limitée à la récolte d'échantillons d'histoire naturelle. Elle s'oriente vers l'instrumentation. Nous avons déjà signalé la difficulté d'obtenir les instruments de physique que La Galissonière voulait faire parvenir à Lotbinière. "Vous aurés a ce que j'espere un

47 Ibid., 365.

48 La Galissonière à Abraham Gradis, Paris, 4 mars 1754. Claude de Bonnault, éd., "Les Archives de la famille Gradis et le Canada », Rapport de l'Archiviste de la Province de Québec pour 1944-1945, 278.

49 « Je vous fais mille remerciemens, Monsieur, des soins que vous avez bien voulu vous donner pour l'envoi en Canada des semences d'arbres fruitiers qui $\mathrm{y}$ manquent et je ne doute pas qu'avec les précautions que vous avez prises, elles n'y réussissent à merveille. Mais je suis fâché que vous ne m'ayés rien dit des petits frais que cela vous a occasionné, dont il n'est pas juste que vous restiés chargé, ainsi que je vous l'ai desjà mandé cy-devant, et d'autant plus que tous les ans, je compte faire des envois pareils ou plus considérables, jusqu'à ce que je sache que les premiers soient arrivés assés à propos pour avoir produit des arbres. $>I d$. à Id., Paris, 29 mars 1751 , Ibid., 278.

50 Id. à Id., Toulon, 20 mai 1754, Ibid., 281. 
micrometre mais je ne sai quand viendra le quart de Cercle $», 51$ écrit La Galissonière à l'ingénieur du roi. Plusieurs années auparavant, il avait fait part à Duhamel du Monceau de son intention d'acheter, ce qui était probablement un microscope, «deux loupes montées ensemble avec un étui $\gg .^{52}$

Lors du voyage du Héros à Louisbourg, à partir de l'Ile d'Aix, lundi le 10 juin 1737, jusqu'au retour au même port, dimanche le $1^{\text {er }}$ décembre 1737 , le ministre de la Marine, Maurepas, avait confié au commandant de vaisseau, La Galissonière, la mission de vérifier « un nouvel instrument pour prendre [la] hauteur ». Les relevés d'observations de latitudes obtenus grâce à l'arbalestrille et au «nouvel instrument» démontrent que l'on ne s'est pas contenté de quelques indications pour fin de vérification. Compte tenu des difficultés initiales de mise au point, on a recueilli ce qui semble être le plus grand nombre d'observations possibles en ce cas. Barrin de La Galissonière accorde le crédit à $M$. de Laiguille, «très bon officier et très exact observateur $\gg$; il suggère une amélioration pratique de cet instrument qui semble bien être un octant dont il est d'avis de généraliser l'emploi dans la marine. ${ }^{53}$ Le 17 mars 1739, il cherchait à obtenir un instrument pour prendre [la] hauteur, pareil à celui qu'il avait deux ans auparavant, et « rectifié suivant les observations que l'on y a faites $\gg .^{54}$

C'est avec soin que Barrin de La Galissonière s'occupe des instruments. "Je croi qu'il est bon de voir revenir le quart de cercle de M. de Cassini et je crains beaucoup que M. de la Cardonie n'ait négligé les précautions nécessaires à sa conservation. Quand on se marie, on ne peut pas songer à tant de choses $\gg .55$

La Galissonière recherche la précision des mesures. « Je vous prie de vérifier l'instrument dont vous vous êtes servi, écritil à Lotbinière, afin que s'il a occasionné quelques autres erreurs

51 La Galissonière à Lotbinière, Paris, 17 mars 1755. APC. Papiers de Lotbinière, No 27-105, 2, 1753-1759, M. G. 18, K 3, 2 (2).

52 Id. à Duhamel du Monceau, Rochefort, 17 mars 1739. APC. (M. G. 18, G. 11), 2.

53 Journal de bord du Héros.

54 La Galissonière à Duhamel du Monceau, Rochefort, 17 mars 1739. APC. (M. G. 18, G. 11), 2.

55 Id. à Id., Toulon, $1^{\text {er }}$ septembre 1756, Ibid., 31. 
que celles qui vous ont été alors connues, on puisse en tenir compte ${ }^{56}$ La nécessité d'instituer des expériences dirigées est mise en lumière dans le seul texte ${ }^{57}$ de La Galissonière qu'il me fut possible de retracer à ce sujet.

Barrin de La Galissonière avoue que sa carrière de marin l'empêche de développer ses connaissances botaniques. ${ }^{58}$ Un délai dans la transmission d'un compte-rendu d'une observation astronomique de Lotbinière a été occasionné par un voyage de Barrin de La Galissonière. «Je n'ai point entendu parler, écrit Pierre Bouguer à Lotbinière, des observations que vous avez envoyées à $M$. le comte de la Galissonière; mais il se peut faire qu'il n'ait pas encore eu le temps de s'arranger à cause du peu de temps qu'il y a depuis son retour $\gg{ }^{59}$ Il n'existe aucun document sur La Galissonière aux Archives de l'Observatoire de Paris, et la correspondance de Bouguer, qui est assez nombreuse, ne contient pas de lettres qui lui soient adressées. ${ }^{60}$

Lotbinière s'était mérité les félicitations de l'astronome Bouguer au sujet de ses remarques sur «l'instrument de M. Hadley ${ }^{61}$ Cet instrument appelé aussi nouveau quartier anglais de

56 Id. à Lotbinière, Paris, 19 février 1754. APC. Papiers de Lotbinière, No 27-105, 2, 1753-1759, M. G. 18, K 3, 2 (2).

57 Il est utile d'en citer un extrait bien qu'il ne soit pas relié à l'activité scientifique de La Galissonière au Canada. "Voici donc ce que je recommanderois en attendant que l'expérience nous en découvre davantage: $1^{\circ}$ qu'on ne recueillit la graine de plane que dans le tems qu'elle est prête à tomber d'elle-mesme, c'est-à-dire au mois d'avril à Constantinople, $2^{\circ}$ qu'on en recueillit une très grande quantité, car ce n'est qu'ainsi qu'on peut se flatter d'en établir un peu en France en la partageant à plusieurs personnes, $3^{\circ}$ que quand elle seroit amassée et bien séchée, on en fît trois lots: le $1^{\text {er }}$ seroit laissé en grosses boules, comme l'arbre les produit; le $2^{\mathrm{e}}$ seroit nettoié de son boutre et mis dans un sac bien sèchement; le $3^{\mathbf{e}}$ seroit seulement froissé et meslé dans une caisse avec de la terre humide; le 4 e seroit meslé de la mesme manière dans de la mousse de forêt dont on auroit bien secoué la terre. Il y aura du malheur si quelqu'une de ces façons ne réussit pas. » La Galissonière à Duhamel du Monceau, Toulon, 4 avril 1743. APC. (M. G. 18, G. 11), 23.

58 Id. à Id., Brest, 14 mai 1744. Ibid., 25.

59 Paris, 22 janvier 1755. APC. Papiers de Lotbinière, No 27-105, 2, 1753-1759, M. G. 18, K 3, 2 (2).

$60 \mathrm{Ce}$ renseignement nous a été aimablement communiqué par M. Jacques Lévy, astronome à l'Observatoire de Paris et historien des sciences.

61 «Ces remarques étoient d'autant plus précieuses pour moi, qu'elles étoient excellentes ... 》. Bouguer à Lotbinière, Paris, 22 janvier 1755. APC. Papiers de Lotbinière, No 27-105, 2, 1753-1759, M. G. 18, K 3, 2 (2). 
réflexion ou octant de $M$. Hadley ${ }^{62}$ fut construit en série en Angleterre et en Hollande, à partir de 1736. En France, le fabricant d'instruments Pierre Lemaire avait pour enseigne $\mathrm{Au} \mathrm{Nou-}$ veau Quartier Anglois. En 1736, un octant de Hadley était à la disposition de Après de Mannevillette qui a rédigé un ouvrage datant de 1739. En raison des difficultés pour l'établissement de bons miroirs, très peu d'instruments de précision ont existé avant 1775.

Le mémoire de Lotbinière relatif à une observation d'éclipse de lune, à Québec, le $1^{\text {er }}$ octobre 1754 , porte une annotation de Pierre Bouguer dont voici un extrait: «il y a donc icy quelque omission essentielle qui empêche de vérifier si l'observateur a eu exactement l'heure vraie $\gg^{63} \mathrm{La}$ Galissonière exige une présentation méthodique des résultats. Il a fait copier les observations de Lotbinière dont il est mécontent et il en a distribué des copies aux membres de l'Académie Royale des Sciences. C'est avec fermeté qu'il fait cette mise au point: «je vous avoue que les originaux qui étoient sur de petits morceaux de papier détachés les uns des autres n'étoient pas trop montrables, et vous me ferés plaisir de me les envoier à l'avenir en cahiers avec des marges et avec un extérieur qui annonce un peu l'attention avec laquelle les observations ont été faites et rédigées. ${ }^{64}$

A l'occasion de la mission scientifique de Léry à Détroit et de celle de Lotbinière à Michilimakinac, les mémoires d'instructions de La Galissonière, le 26 mai 1749 et le 19 juin 1749, portent sur des questions intéressant l'histoire naturelle, l'astronomie, la géographie et les ressources naturelles dont les observations doivent être consignées avec exactitude. ${ }^{65}$ « Il [de Léry] fera un journal exact qu'il commencera le jour de son départ et ne finira qu'à celuy de son arrivée. 》 L'instruction du $\mathrm{N}^{\circ}$ XXI

62 \& Décrit par Hooke dès 1666, puis par Newton qui ne publia pas son travail, le sextant, d'abord appelé octant, a été réalisé d'une façon pratique par Hadley en 1731 ». Pierre Humbert, «L'astronomie de la Renaissance à nos jours 》, Histoire de la science, publiée sous la direction de Maurice Daumas (Paris, 1957), 745-746.

2 (2).

63 APC. Papiers de Lotbinière, No 27-105, 1753-1759, M. G. 18, K. 3,

64 La Galissonière à Lotbinière, Paris, 17 mars 1755 . Ibid.

65 Instruction pour le S. de Léry. Archives de la Province de Québec. 
du mémoire adressé à de Léry est similaire à celle du $\mathrm{N}^{\circ}$ XXIII de l'Instruction pour le S. de Lotbinière: ${ }^{66}$ " Si chemin faisant il peut ramasser quelques unes des graines et plantes mentionnées dans le mémoire qui luy sera remis avec ceci, il les apportera, mais cela ne doit point le détourner des objets plus interessans pour le service. »

En $1750,{ }^{67}$ Barrin de La Galissonière était nommé directeur du Dépôt de la Marine (ancienne appellation du Service Hydrographique de la Marine) avec le titre de «Préposé à l'examen et à la garde des plans, cartes, journaux et mémoires de la Marine ${ }^{68} \mathrm{Il}$ avait préparé des missions scientifiques au Canada. Il organisera plus tard les missions de Joseph-Bernard Chabert de Cogolin en Amérique du Nord, de Gabriel Bory sur les côtes d'Espagne et de Nicolas-Louis de Lacaille au Cap de Bonne-Espérance.

Les échanges scientifiques entre la France, l'Angleterre et les colonies britanniques de l'Amérique du Nord ne furent pas rompus par les guerres qui ont opposé les deux métropoles. ${ }^{69}$ De nombreux naturalistes français ont exploré les régions nordest et sud-est des futurs États-Unis, au XVIII ${ }^{\mathrm{e}}$ siècle. ${ }^{70}$

La correspondance du gouverneur intérimaire, Barrin de La Galissonière, avec le gouverneur de New York, George Clinton, porte sur des questions militaires. ${ }^{71}$ Une lettre de La Galissonière

66 Québec, 19 juin 1749. APC. Papiers de Lotbinière, No 1-25, 1, 16631753, M. G. 18, K 3, 2 (1).

67 Bibliothèque Nationale de Paris. Le manuscrit des Nouvelles acquisitions françaises No $9492, \mathrm{p} .246$, indique la date de nomination au $1^{\text {er }}$ janvier 1750. La date du $1^{\text {er }}$ février 1750 est rapportée dans les ouvrages suivants: L. Lejeune, Dictionnaire général du Canada (2 vol., Ottawa, 1931), 2: 35 et S. L., Notice biographique sur La Galissonière et les bâtiments ayant porté son nom. Service historique de l'Etat-Major général du Ministère des Armées ( $\mathrm{s}$. d.), 1.

68 Ce renseignement a été obtenu grâce à l'obligeance de M. l'Ingénieur Général Gougenheim, directeur du Service Hydrographique de la Marine.

69 Jean Jacquot, Le Naturaliste Sir Hans Sloane, 1660-1753, et les échanges scientifiques entre la France et l'Angleterre. Les Conférences du Palais de la Découverte. Série D, No 25. Université de Paris. (Alençon, 1954), 8. 41-51.

70 Les Botanistes français en Amérique du Nord avant 1850, 17-40;

${ }^{71}$ British Museum, Mss. 32818: 21, 30, 37, 41, 61. 
au naturaliste Lemonnier contient une indication relative à l'obtention de variétés d'arbres de la colonie britannique de Virginie. La Galissonière s'enquiert auprès de Lemonnier si l'on a reçu les graines qu'il a remises au suisse de l'Hôtel de Noailles. "C'étoit presque tous arbres de Virginie et il y en avoit d'interessans, je vous en donnois avis dans le mesme tems et j'adressai ma lettre qui étoit Contresignée Rouillé à St. Germain, mais je n'ai pas eu de reponse. ${ }^{72}$

La contribution scientifique de La Galissonière n'est pas limitée à la période d'administration coloniale. Le gouverneur intérimaire atteignait Québec, le 19 septembre $1747 ;^{73}$ il devait quitter le Canada peu après l'arrivée de Pierre-Jacques Taffanel, marquis de La Jonquière, qui venait prendre possession de son poste, le 15 août 1749. Nous avons vu que, dès 1739 , Barrin de La Galissonière avait proposé à Duhamel du Monceau de venir en aide à un botaniste canadien, l'abbé Gosselin. En 1741, tout en tenant compte de la politique mercantiliste de la métropole, il recommandait d'employer les richesses forestières de la colonie à la construction navale que Jean Talon avait inaugurée au Canada. "Tout ce qu'on pourroit faire ce seroit d'engager les marchands par quelque gratification et par l'espérance de la durée des vaisseaux a y faire bâtir cela fortifieroit le pays et le Roi par la suitte pourroit profiter pour luy mesme des ouvriers qu'ils auroient dressés. $\gg 75$

$\mathrm{Au}$ début de sa période d'administration de la NouvelleFrance, La Galissonière insiste sur la nécessité de consolider la colonie. ${ }^{76}$ Plus tard, en 1751, il affirmera «qu'il est de la dernière importance et d'une nécessité absolue de n'omettre aucun moyen

72 Paris, $1^{\mathrm{er}}$ octobre 1752. Bibliothèque centrale du Muséum national d'histoire naturelle.

73 Hocquart à Maurepas, Québec, 26 septembre 1747. APC. Transcription tirée des Archives des Colonies, série C11A, 88: 39. 424-425

${ }^{74}$ Guy Frégault, Le Grand Marquis, Pierre de Rigaud de Vaudreuil,

75 Lettre autographe signée à Duhamel du Monceau. A bord du Tigre, en rade de Toulon, 7-8 octobre 1741. Bibliothèque centrale du Muséum national d'histoire naturelle.

${ }_{76} \mathrm{La}$ Galissonière à Maurepas, Québec, 24 octobre 1747. APC. AC, C11A, 87-2: 204-210. 
et de n'épargner aucune dépense pour assurer la conservation du Canada .... ${ }^{77}$

L'activité scientifique de La Galissonière n'était pas seulement dirigée vers la recherche désintéressée. C'est Pierre Kalm qui rapporte que le gouverneur lui a indiqué plusieurs moyens d'utiliser l'histoire naturelle à des fins politiques. ${ }^{78}$ La contribution scientifique de Barrin de La Galissonière au Canada s'intégrait à une œuvre d'expansion coloniale.

Roland LamontagNe, Ph.D.

Faculté des Arts,

Université de Montréal.

77 1751. Mémoire sur les Colonies de la France dans l'Amérique septentrionale. Par M. le Marquis de La Galissonière ». Affaires Etrangères. Transcription des APC. M. G. 5, B-1 (22). Mémoires et Documents. Amérique, 24-2: 190. ${ }_{78}$ P. Kalm, Travels into North America, 2: 369. 